Tecno Lógicas

ISSN 0123-7799

Vol. 19, No. 36, pp. 27-40

Enero-junio de 2016

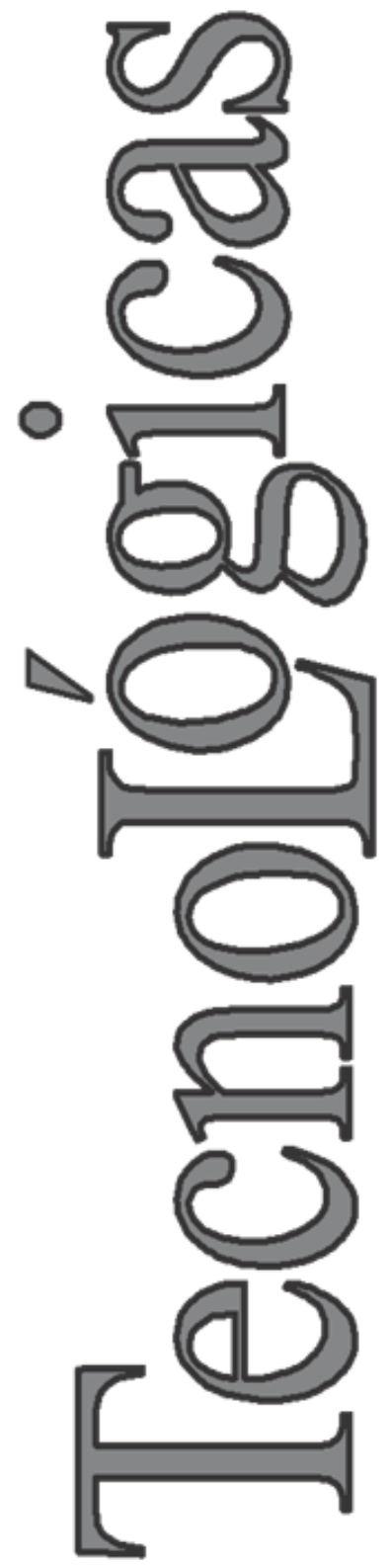

(C) Copyright 2015 por autores y Tecno Lógicas Este trabajo está licenciado bajo una Licencia Internacional Creative Commons Atribución (CC BY)

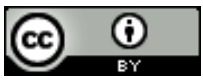

\section{Microestructura y resistencia adhesivo-cohesiva de recubrimientos de níquel, carburo de tungsteno y óxido de cromo aplicados por aspersión térmica}

\author{
Microstructure and adhesive-cohesive \\ strenght of nickel, tungsten carbide and \\ chromium oxide thermal spray coatings
}

Juan F. Santa-Marín ${ }^{1}$ y Alejandro Toro-Betancur ${ }^{2}$

Recibido: 14 de abril de 2015,

Aceptado: 14 de octubre de 2015

Cómo citar / How to cite

J.F. Santa-Marín, y A. Toro-Betancur, "Microestructura y resistencia adhesivo-cohesiva de recubrimientos de níquel, carburo de tungsteno y óxido de cromo aplicados por aspersión térmica”, Tecno Lógicas, vol. 19, no. 36, pp. 27-40, 2016.

1 Ph.D. en Ciencia y Tecnología de los Materiales, Grupo de Investigación Materiales Avanzados y Energía- MATyER, Instituto Tecnológico Metropolitano, Grupo de Tribología y Superficies, Universidad Nacional de Colombia, Medellín-Colombia, juansanta@itm.edu.co

2 Ph.D. Ingeniería Metalúrgica, Grupo de Tribología y Superficies, Universidad Nacional de Colombia , Sede Medellín, MedellínColombia, aotoro@unal.edu.co 


\section{Resumen}

Se aplicaron cuatro recubrimientos por aspersión térmica por combustión con material de aporte en polvo: dos aleaciones ricas en níquel, un recubrimiento de óxido de cromo y un recubrimiento rico en carburos de tungsteno y partículas de Ni-Cr, sobre acero inoxidable ASTM A743 grado CA6NM. Se estudió la microestructura de los depósitos y la variación en la resistencia adhesivo-cohesiva de las capas de acuerdo con la norma ASTM C633, en función de los parámetros de preparación de la superficie (temperatura de precalentamiento y rugosidad superficial). La caracterización microestructural fue realizada utilizando difracción de rayos X (DRX) y microscopía óptica (MO) y electrónica de barrido (MEB), las mediciones de dureza fueron realizadas de acuerdo con la norma ASTM E384. Los ensayos de resistencia adhesivo-cohesiva fueron realizados bajo norma ASTM C633. Los recubrimientos presentaron microestructuras con niveles de defectos del orden de $8 \%$ para capas base y entre 15 y $30 \%$ para capas cerámicas, las cuales son aceptables para la técnica empleada. En todos los casos se obtuvieron espesores de capas homogéneos del orden de $0.5 \mathrm{~mm}$. La resistencia adhesivo-cohesiva alcanzó entre 10 y $19 \mathrm{MPa}$ para la mayoría de las capas. Los valores alcanzados de adherencia fueron satisfactorios en la mayoría de los casos y, en términos generales, la resistencia adhesivo-cohesiva incrementó significativamente (mayor al 25\%) al modificar los parámetros de preparación de las superficies especialmente para el recubrimiento $\mathrm{WC} / \mathrm{Co}-\mathrm{Ni}$.

\section{Palabras clave}

Recubrimientos por aspersión térmica, acero inoxidable, resistencia adhesivocohesiva, microestructura.

\section{Abstract}

Four thermal spray coatings were applied by oxy-fuel process onto ASTM A743 CA6NM (13Cr-4Ni) steel, namely: two Ni-rich alloys, a chromium oxide and a combined tungsten carbide/Ni-Cr particles coating. The microstructure and adhesive/cohesive strength of the coatings were studied and related to the surface preparation parameters (preheating temperature and surface roughness). The microstructures were studied by XRay diffraction (XRD), scanning electron (SEM) and light optical (LOM) microscopy and hardness was measured according to ASTM E384 standard. The adhesive/cohesive strength was measured according to ASTM C633 standard. The microstructure of the coatings showed porosity ( $8 \%$ for bond coatings and from 15 to $30 \%$ for ceramic coatings) within acceptable values for flame spraying Homogeneous coating thicknesses of circa $0.5 \mathrm{~mm}$ were obtained. Adhesive strength was among 10 to $19 \mathrm{MPa}$ for most of coatings. The coating-substrate adhesive strength was satisfactory in most cases and significant variations (higher than 25\%) in adhesive/cohesive strength were induced by changes in surface preparation parameters, especially in the case of $\mathrm{WC} / \mathrm{Co}-\mathrm{Ni}$ coating.

\section{Keywords}

Thermal Spray Coatings, Stainless Steel, Adhesive-cohesive Strength, Microstructure. 

por aspersión térmica

\section{INTRODUCCIÓN}

El término aspersión térmica describe una familia de procesos que usan energía generada químicamente o eléctricamente para fundir o reblandecer y acelerar partículas o gotas de un material que son depositadas sobre una superficie [1]. Estos materiales pueden ser metálicos, poliméricos, cerámicos o compuestos, lo cual representa una ventaja significativa desde el punto de vista de mejora de propiedades de superficie de diversos elementos de máquinas. Debido a las características inherentes a los procesos de formación de las capas, la principal limitación de estos recubrimientos es la resistencia adhesivo-cohesiva de las capas depositadas, la cual alcanza valores entre 15 y $20 \mathrm{MPa}$ para la técnica de aspersión por combustión sobre aceros al carbono [1]. Adicionalmente, se debe notar que los parámetros de preparación de la superficie tienen una gran influencia en los valores de adherencia como los muestran diversos estudios [2]-[4].

Existen en la literatura varios estudios [5]-[7] que relacionan la rugosidad de la superficie con la adherencia de los recubrimientos. En el estudio de Salinas et al. [5] se observa que, a niveles mayores del parámetro $\mathrm{Ra}$, la adherencia se incrementa en 0,4 MPa para multicapas y en 0,5 $\mathrm{MPa}$ para capas de anclaje en procesos de aspersión por combustión. Por otro lado, para el estudio realizado por Paredes y coautores [6], la resistencia se reduce en $2 \mathrm{MPa}$ aproximadamente con el incremento de la rugosidad Ry (media aritmética de los 5 valores sucesivos de rugosidad máxima a lo largo de la longitud de medición) del sustrato. Finalmente, los resultados de Mellali et al. [7] mostraron que un incremento en Ra incrementa la adhesión de los recubrimientos hasta en $10 \mathrm{MPa}$ para el proceso de plasma en aire. Como se puede observar, aún no existe un consenso sobre el efecto de la rugosidad en la resistencia adhesivo-cohesiva de los recubrimientos.
Por otro lado, varios autores [5], [7], [8] han estudiado el efecto de la temperatura de precalentamiento en la adhesión de recubrimientos. El trabajo de Salinas et al. [5] mostró cambios en la adhesión de hasta $15 \mathrm{MPa}$ para procesos de aspersión por combustión para recubrimientos de $\mathrm{Al}_{2} \mathrm{O}_{3}$ con temperaturas de $120^{\circ} \mathrm{C}$. Mellali et al. [7] mostraron que al precalentar a $330{ }^{\circ} \mathrm{C}$ se incrementa la adhesión en $22 \mathrm{MPa}$ para recubrimientos sobre acero al carbono. Fauchais et al. encontraron que un aumento de $100^{\circ} \mathrm{C}$ incrementa la adherencia en $20 \mathrm{MPa}$. Sin embargo, se debe anotar que estos autores no han estudiado varios recubrimientos ni recubrimientos de Tungsteno.

Otros autores [9] han estudiado el efecto de los parámetros de la preparación de la superficie en la rugosidad de los sustratos, pero no han correlacionado los resultados de rugosidad con la resistencia adhesivo-cohesiva de los recubrimientos. En el trabajo de Nistal y colaboradores [9] se evaluó el efecto del tiempo de aplicación del impacto de arena sobre los parámetros Ra (Rugosidad media) y Ry (Rugosidad media máxima). Estos autores encontraron que después de 20 segundos de aplicación del impacto de la arena, la rugosidad se incrementa significativamente dependiendo de la presión para la aplicación de recubrimientos de aspersión térmica mediante el proceso de aspersión térmica por llama. Recientemente, otros autores [10] han estudiado la resistencia adhesivo cohesiva de recubrimientos de zirconia estabilizada con itrio por aspersión por plasma atmosférico media, mediante la norma ASTM C633. Los autores encontraron valores cercanos a los $50 \mathrm{MPa}$ con desviaciones estándar del orden de 10 a $15 \mathrm{MPa}$. Lo anterior indica que los recubrimientos por esta técnica poseen una resistencia superior a los recubrimientos obtenidos por la técnica de aspersión por llama y, adicionalmente, encontraron que la microestructura posee un efecto significativo en la 
resistencia adhesivo-cohesiva de los recubrimientos.

De acuerdo con lo anterior, en este trabajo se genera apropiación de conocimiento sobre los procedimientos de aplicación de recubrimientos por aspersión térmica, y se realiza un estudio sobre el efecto de la modificación de los parámetros de preparación de la superficie en la resistencia adhesivo-cohesiva de los recubrimientos bajo la norma ASTM C633.

\section{METOdOLOGíA}

\subsection{Materiales}

Se aplicaron recubrimientos mediante la técnica de aspersión por llama con material de aporte en polvo $(O F P)$, utilizando un equipo Terodyn 2000 (Eutectic Castolin) sobre acero inoxidable martensítico ASTM A743, Tipo CA6NM (13Cr-4Ni) homogenizado a $1050^{\circ} \mathrm{C}$ durante 2 horas y revenido a $620^{\circ} \mathrm{C}$ durante dos horas y enfriado al aire. Se utilizaron como base las recomendaciones del fabricante realizando modificaciones al procedimiento de aplicación de cada tipo de recubrimiento. Se utilizó como capa base en todos los casos una aleación de base níquel $\left(\mathrm{Ni}^{-1}\right)$. Los parámetros de aplicación utilizados en el proceso se observan en la Tabla 1. La preparación de las superficies fue realizada mediante impacto de arena (sandblasting) y de óxido de aluminio. Los valores del parámetro $\mathrm{Ra}$ (medidos mediante rugosímetro Mitutoyo SJ 201) fueron controlados entre 5,4 y $6,4 \mu \mathrm{m} \mathrm{me}^{-}$ diante la modificación del abrasivo con el cual se realizó la preparación de la superficie. Los dos abrasivos utilizados fueron sílice con granulometría AFS 20/40 y alúmina con granulometría AFS 20. Los valores de los parámetros utilizados para la preparación de las superficies se presentan en la Tabla 2.

Tabla 1. Parámetros de aplicación de los recubrimientos. Fuente: Autores

\begin{tabular}{|c|c|c|c|c|c|c|c|}
\hline Recubrimiento & Tipo de material de aporte & $\begin{array}{l}\text { Caudales } \\
\text { reales } \\
\text { /cfm }\end{array}$ & $\begin{array}{c}\text { Presión } \\
\text { Aire } \\
\text { / psi }\end{array}$ & $\begin{array}{l}\text { Posición } \\
\text { válvula T2 }\end{array}$ & $\begin{array}{c}\text { Distancia } \\
\text { de aplica- } \\
\text { ción } \\
/ \mathrm{mm} \\
\end{array}$ & $\begin{array}{c}\text { Blanco de } \\
\text { aspersión } \\
\text { /mm }\end{array}$ & $\begin{array}{l}\text { Velocidad de } \\
\text { aplicación } \\
\text { / } \mathrm{cm} \mathrm{s}^{-1}\end{array}$ \\
\hline $\mathrm{Ni}-1$ & $\begin{array}{l}\text { Partículas ricas en } \\
\mathrm{Ni}(95 \%) \text { y Al }(5 \%)\end{array}$ & $\begin{array}{c}\mathrm{O}_{2}=35 \\
\mathrm{CH}_{2}=70\end{array}$ & 30 & 9 & 200 & 22 & 10 \\
\hline $\mathrm{Cr}$ & Óxidos de cromo (100\%) & $\begin{array}{c}\mathrm{O}_{2}=40 \\
\mathrm{CH}_{2}=60\end{array}$ & 11 & 10 & 200 & 14 & 8 \\
\hline $\mathrm{Ni}-2$ & $\begin{array}{l}\text { Partículas ricas en } \\
\mathrm{Ni}, \mathrm{Cr}, \mathrm{Mo}, \mathrm{Ti}^{1}\end{array}$ & $\begin{array}{c}\mathrm{O}_{2}=40 \\
\mathrm{CH}_{2}=60\end{array}$ & 35 & 10 & 200 & 27 & 13 \\
\hline $\mathrm{WC} / \mathrm{Co}-\mathrm{Ni}$ & $\begin{array}{l}\text { Partículas de WC/Co (46\%) } \\
\text { y Ni-Fe-Cr }(54 \%)\end{array}$ & $\begin{array}{c}\mathrm{O}_{2}=38 \\
\mathrm{CH}_{2}=60\end{array}$ & 30 & 13 & 75 & 7 & 10 \\
\hline \multicolumn{8}{|c|}{$\begin{array}{l}{ }^{1} \text { Debido al proceso de fabricación de las partículas (cladding) no es confiable reportar una composición química promedio } \\
\text { mediante la técnica utilizada (EDS). } \\
{ }^{2} \text { La posición de la válvula T es un parámetro indicador del equipo que provee información sobre la cantidad de material de } \\
\text { aporte que se está alimentando durante el proceso de aspersión. } \\
\text { Nota: se muestran los valores de los parámetros de aplicación en unidades del sistema inglés debido a que los controles del } \\
\text { equipo operan con base en dicho sistema. }\end{array}$} \\
\hline
\end{tabular}

Tabla 2. Parámetros de preparación de las superficies de los recubrimientos. Fuente: Autores

\begin{tabular}{lccccc}
\hline Tipo de abrasivo & $\begin{array}{c}\text { Caudal de } \\
\text { aire } / \mathrm{cfm}\end{array}$ & $\begin{array}{c}\text { Presión de } \\
\text { aire/ KPa }\end{array}$ & $\begin{array}{c}\text { Distancia de } \\
\text { aplicación } / \mathrm{mm}\end{array}$ & $\begin{array}{c}\text { Tiempo de } \\
\text { aplicación /s }\end{array}$ & $\begin{array}{c}\text { Granulometría } \\
\text { AFS }\end{array}$ \\
\hline Óxido de silicio & 13,5 & 7,5 & 10 & 15 & $20 / 40$ \\
\hline Óxido de aluminio & 13,5 & 7,5 & 10 & 15 & 20 \\
\hline
\end{tabular}


Se estudiaron dos temperaturas de precalentamiento denominadas baja y alta, las cuales se alcanzaron mediante el uso controlado de la llama suministrada por el equipo de aspersión y mediante el control de la temperatura inter-pase durante la aplicación de los recubrimientos.

\subsection{Caracterización microestructural y quí- mica}

La caracterización microestructural se realizó usando microscopio electrónico de barrido (MEB) JEOL 5910LV con microsonda EDS acoplada y microscopio óptico (MO) Nikkon PME. Igualmente se obtuvieron difractogramas de rayos $\mathrm{X}$ de los materiales de aporte y de los recubrimientos obtenidos utilizando un difractómetro X'Pert Pro, con radiación incidente de $\mathrm{Cu}$, detector PIXCEL y software Xpert High Score Plus utilizando la base de datos PDF2, 2006.

La estimación de las características microestructurales de los recubrimientos fue realizada mediante análisis digital de imágenes sobre las micrografías obtenidas mediante MEB y MO. Se realizaron mediciones de dureza en un durómetro marca Wolpert en escala Vickers y un microdurómetro en escala Vickers, utilizando diferentes valores de carga y un tiempo de aplicación fijo de 15s. Las mediciones se llevaron a cabo siguiendo los lineamientos establecidos por la norma ASTM E384.

\subsection{Ensayos de resistencia adhesivo- cohesiva}

Los ensayos de resistencia adhesivocohesiva fueron realizados de acuerdo con la norma ASTM C633-01 [11], en máquina de tracción de eje horizontal con capacidad máxima de 2 Ton, la cual se muestra en la Fig. 1a. Cada muestra ensayada estaba constituida por dos cilindros de $25 \mathrm{~mm}$ de diámetro, uno de ellos denominado cuerpo, sobre el cual fueron aplicadas las capas, y otro sin recubrir llamado contracuerpo. Los recubrimientos fueron depositados de acuerdo con el procedimiento descrito en 2.1. Una vez aplicados los recubrimientos, se rectificaron las superficies de los cuerpos para garantizar una superficie plana y perpendicular al eje del cilindro y un espesor de capa igual en todos los casos de 500 $\mu \mathrm{m}$. Ambos cilindros (cuerpo y contracuerpo) fueron unidos mediante un adhesivo epóxico con una resistencia teórica de 30 $\mathrm{MPa}$ utilizando un dispositivo de alineación que se muestra en la Fig. 1b, y se les proporcionó un tiempo de curado de 3 días. Al realizar el ensayo se aplicó una carga de tensión a una velocidad constante de 0.013 $\mathrm{mms}^{-1}$ hasta que se presentó la falla. Se reportaron los valores de carga de ruptura y se caracterizaron las superficies de falla de acuerdo con los indicativos de la norma ASTM C633 que se ilustran en la Fig. 1c.

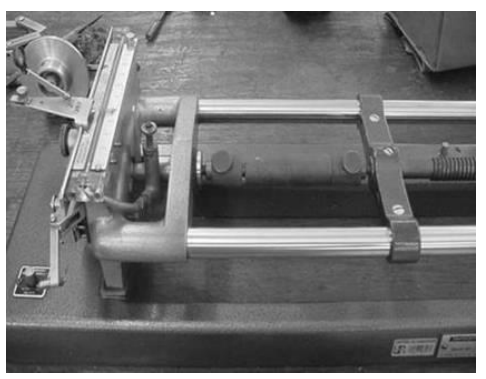

(a)

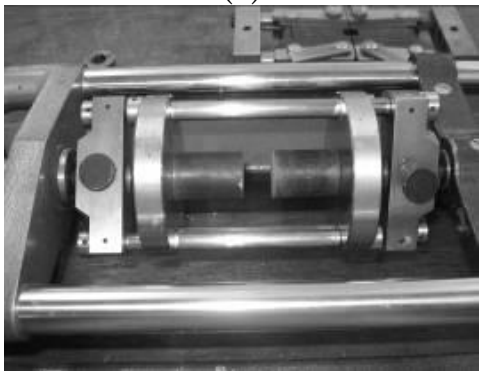

(b)

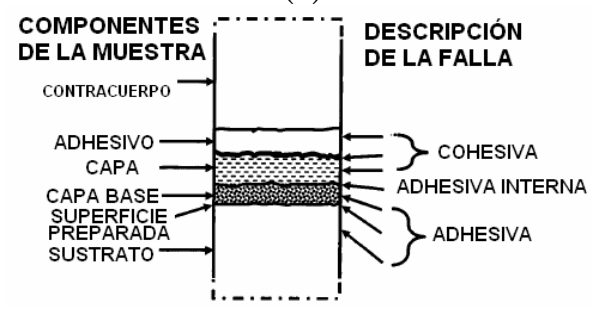

(c)

Fig. 1. Características de ensayos de resistencia adhesivocohesiva realizados bajo norma ASTM C633. Fuente: Autores 
Adicionalmente, se realizaron observaciones de las superficies de falla mediante MEB con el fin de identificar las características microestructurales de las mismas.

\section{RESULTADOS Y DISCUSIÓN}

\subsection{Caracterización microestructural}

Las observaciones metalográficas del acero utilizado como substrato reportaron una microestructura martensítica con morfología de listones, típica de aceros de bajo carbono, como se muestra en la Fig. 2. La dureza media del acero es $280 \mathrm{HV}_{31.25}$. De acuerdo con la literatura, este valor de dureza es consecuente con la historia térmica del material [12], [13]. Imágenes generales de las superficies preparadas y los valores de parámetros de rugosidad superficial promedio ( $\mathrm{Ra}$ ) y rugosidad RMS (Rq) obtenidos mediante el proceso de adecuación de las superficies previo a la aplicación se presentan en la Fig. 3. Allí se observa que las superficies son más rugosas al ser preparadas con partículas de óxido de aluminio, lo cual es consistente con la mayor angulosidad de este tipo de abrasivo al ser comparado con el óxido de silicio. La dureza de los abrasivos presenta un efecto significativo en la rugosidad dado que influye sobre la magnitud del desgaste erosivo artificial que se realiza durante el impacto de arena (sandblasting). Para el caso de este estudio, las partículas de óxido de silicio poseen una dureza del orden de 800 a $900 \mathrm{HV}$, mientras que las partículas de alúmina alcanzaron valores entre 1400 y $1600 \mathrm{HV}$. De acuerdo con lo anterior, es posible generar mayor desgaste erosivo artificial (y por lo tanto mayor rugosidad superficial para el caso específico) con las partículas de alúmina.

La microestructura de los recubrimientos utilizados se muestra en la Fig. 4. El recubrimiento $\mathrm{Ni}-1$ (Fig. 4a) está compuesto de una matriz de níquel fase $\gamma^{\prime}$ (ver difractograma de rayos $\mathrm{X}$ en Fig. 5) con dureza media de $191 \mathrm{HV}_{300 \mathrm{~g}, 15 \mathrm{seg}} \mathrm{y}$ un porcentaje del $15 \%$ de óxidos de aluminio con dureza media $1533 \mathrm{HV}_{25 \mathrm{gf}, 15}$. El porcentaje de porosidad estimado es $8 \%$.

El recubrimiento $\mathrm{Ni}^{-} 2$ está compuesto de una matriz rica en $\mathrm{Ni}-\mathrm{Fe}-\mathrm{Mo}-\mathrm{Si}$ con dureza media de $385 \mathrm{HV}_{50 \mathrm{gf}, 15}$ identificada como (1), en la Fig. 4b y de un $28 \%$ de partículas de óxido de titanio con dureza media de $701 \mathrm{HV}_{50 \mathrm{gf}, 15}$ identificadas como (2) en la misma figura (partículas más oscuras). El porcentaje de porosidad estimado fue del 8\%. La microestructura del recubrimiento $\mathrm{Cr}$ se observa en la Fig. 4c, consta de una única fase de $\mathrm{Cr}_{2} \mathrm{O}_{3}$ (ver difractograma de rayos $\mathrm{X}$ en Fig. 5c) con dureza media de $1853 \mathrm{HV}_{300 \mathrm{~g}, 15 \mathrm{seg}} \mathrm{y}$ un porcentaje de porosidad del 30\%. En la misma Fig. 4c puede apreciarse también la capa base de aleación $\mathrm{Ni}^{-1}$.

La microestructura del recubrimiento WC/Co-Ni se muestra en la Fig. 4d. Allí se observa la capa de anclaje de aleación $\mathrm{Ni}^{-1}$ identificada como (1) la cual alcanza un espesor del orden de $100 \mu \mathrm{m}$. La capa del recubrimiento $\mathrm{WC} / \mathrm{Co}^{-} \mathrm{Ni}$ consta de fracciones aproximadamente iguales de partículas de WC-Co con una microdureza media

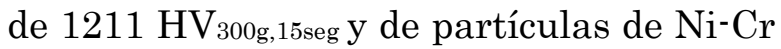
con una microdureza media de 639 $\mathrm{HV}_{300 \mathrm{~g}, 15 \text { seg. }}$ El porcentaje de porosidad del recubrimiento es del orden de $15 \%$. Las fases presentes en este recubrimiento pueden ser observadas en el difractograma de rayos X de la Fig. 5d.

La identificación de las fases de los recubrimientos, previamente descritos, se apoya en los resultados de difracción de rayos X mostrados en la Fig. 5. Allí se observa que los óxidos identificados en los recubrimientos $\mathrm{Ni}^{-1}$ y Ni-2 no poseen alta cristalinidad debido a las altas velocidades de enfriamiento. Los picos identificados corresponden con la matriz rica en níquel de ambos recubrimientos. Este resultado coincide con lo observado por otros autores [14]. Para el caso del recubrimiento $\mathrm{Cr}$ solo se observa una fase $\left(\mathrm{Cr}_{2} \mathrm{O}_{3}\right)$, mientras que el recubrimiento $\mathrm{WC} / \mathrm{Co}-\mathrm{Ni}$ presenta varias 
Microestructura y resistencia adhesivo-cohesiva de recubrimientos de níquel, carburo de tungsteno y óxido de cromo aplicados por aspersión térmica

fases parcialmente amorfas caracterizadas por la presencia de una zona cóncava cercana a los ángulos $2 \theta$ entre 40 y $45^{\circ}$ en los difractogramas correspondientes. La Tabla 3 muestra un resumen de las principales características de los recubrimientos estudiados.

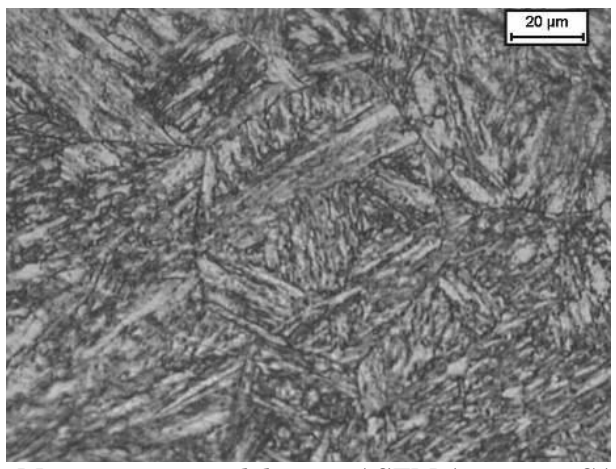

Fig. 2. Microestructura del acero ASTM A743 tipo CA6NM. Ataque Vilella, 540 X (MO). Fuente: Autores

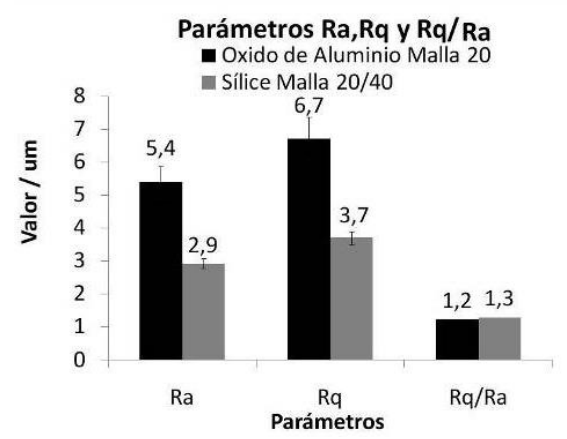

a) Parámetros Ra, Rq y relación entre Rq y Ra

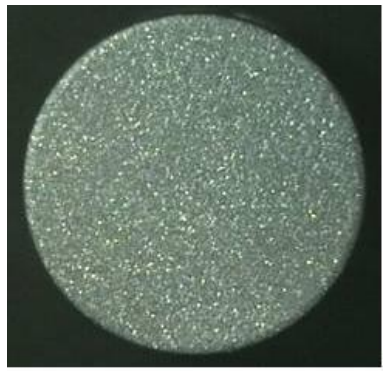

b) Preparación con alúmina

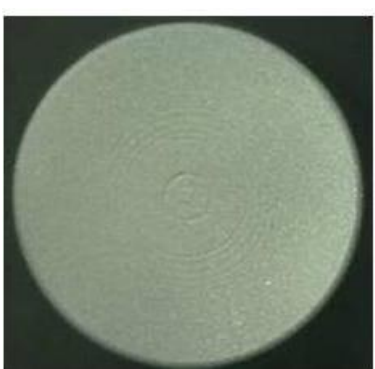

c) Preparación con sílice
Fig. 3. Valor de los parámetros Ra y Rq de las superficies (a) antes de la aplicación e imágenes generales del aspecto de las superficies preparadas (b, c). Fuente: Autores

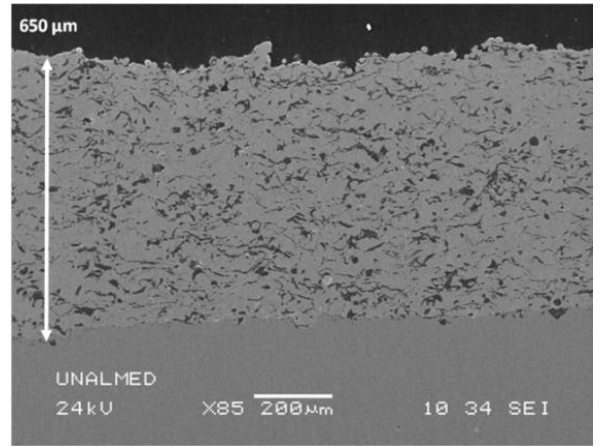

(a)

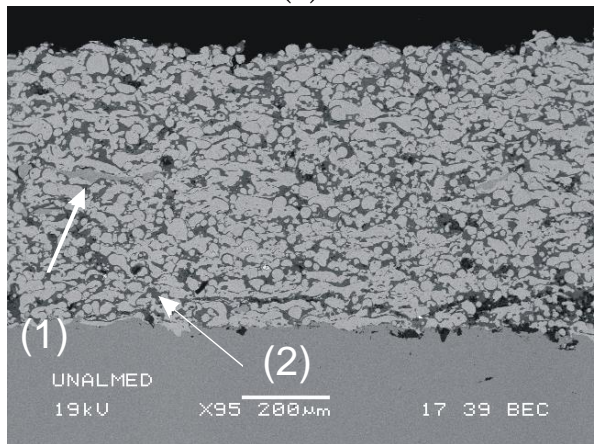

(b)

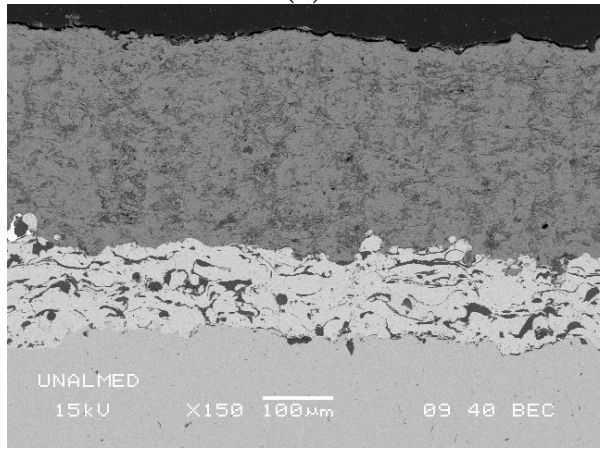

(c)

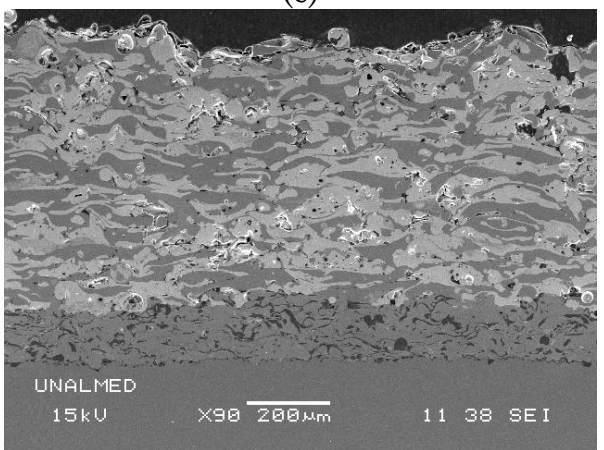

(d)

Fig. 4. Microestructura de recubrimientos obtenidos. Fuente: Autores 


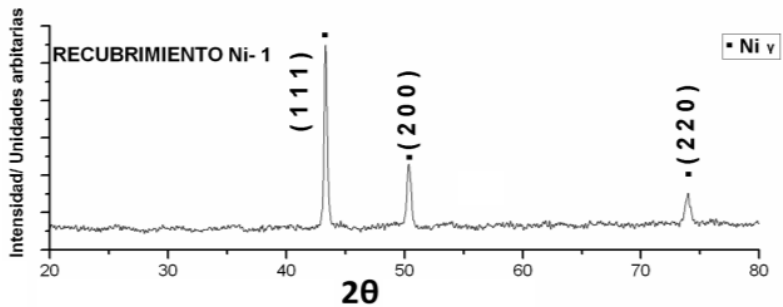

(a)

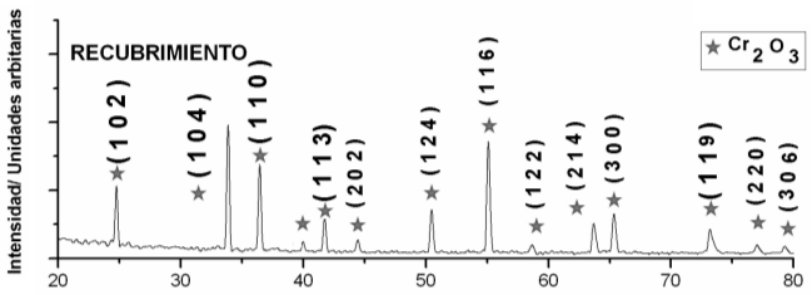

(c)

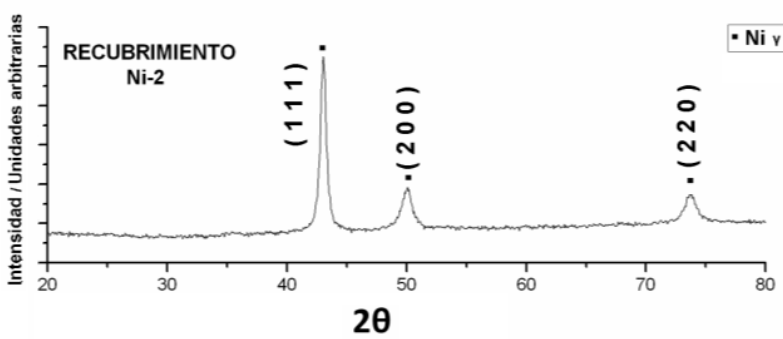

(b)

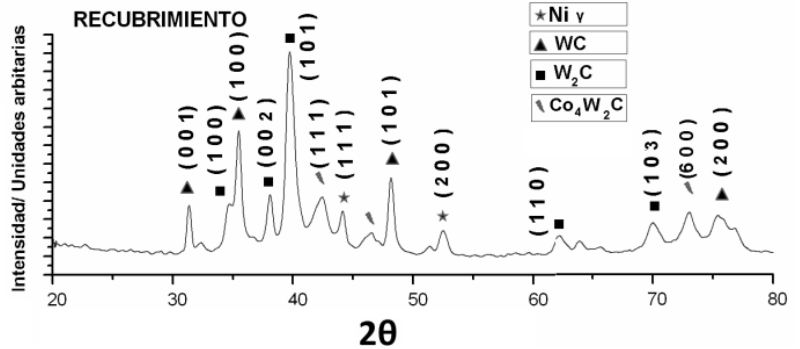

(d)

Fig. 4. Microestructura de recubrimientos obtenidos. Fuente: Autores

Tabla 3. Resumen de características microestructurales de los recubrimientos. Fuente: Autores

\begin{tabular}{|c|c|}
\hline Recubrimiento & Características de las fases \\
\hline $\mathrm{Ni}-1$ & $\begin{array}{l}85 \% \mathrm{Ni}\left(191 \mathrm{HV}_{300 \mathrm{~g}, 15 \mathrm{seg}}\right)+15 \% \\
\mathrm{Al}_{2} \mathrm{O}_{3}\left(1533 \mathrm{HV}_{25 \mathrm{gf}, 15}\right), 8 \% \text { Poros }\end{array}$ \\
\hline $\mathrm{Ni}-2$ & $\begin{array}{c}72 \% \mathrm{Ni}^{-\mathrm{Fe}}-\mathrm{Mo}-\mathrm{Si}\left(385 \mathrm{HV}_{50 \mathrm{gf}, 15}\right)+ \\
28 \% \mathrm{TiO}_{2}\left(701 \mathrm{HV}_{50 \mathrm{gf}, 15}\right), 8 \% \\
\text { Poros }\end{array}$ \\
\hline $\mathrm{WC} / \mathrm{CO}-\mathrm{Ni}$ & $\begin{array}{c}54 \% \mathrm{Ni}-\mathrm{Cr}-\mathrm{Fe}(639 \\
\left.\mathrm{HV}_{300 \mathrm{~g}, 15 \mathrm{seg}}\right)+46 \% \mathrm{WC} / \mathrm{Co}(1211 \\
\left.\mathrm{HV}_{300 \mathrm{~g}, 15 \mathrm{seg}}\right), 15 \% \text { Poros }\end{array}$ \\
\hline $\mathrm{Cr}$ & $\begin{array}{c}100 \% \mathrm{Cr}_{2} \mathrm{O}_{3}\left(1853 \mathrm{HV}_{300 \mathrm{~g}, 15 \mathrm{seg}}\right), 30 \% \\
\text { Poros }\end{array}$ \\
\hline
\end{tabular}

\subsection{Ensayos de resistencia adhesivo- cohesiva}

La Fig. 6 muestra los valores medios y la desviación estándar obtenidos en las pruebas de resistencia adhesivo-cohesiva realizadas de acuerdo con la norma ASTM C633 para todos los recubrimientos estudiados y la variación de los resultados en función del abrasivo usado en la preparación de la superficie y de la temperatura de precalentamiento.

Para el caso del recubrimiento $\mathrm{Ni}-1$, los resultados indican que los mayores valores de resistencia se encuentran para las $\mathrm{su}^{-}$ perficies preparadas mediante el uso de óxido de aluminio y que al incrementar la temperatura de precalentamiento es posible incrementar los valores de resistencia de los recubrimientos. En todos los casos las fallas fueron predominantemente del tipo adhesivo. El efecto del acabado superficial es más pronunciado en comparación con el efecto de la temperatura de precalentamiento.

Los niveles más bajos de carga fueron $14,2 \pm 1,2 \mathrm{MPa}$ y los niveles más altos fueron del orden de 19,3 $\pm 1,0 \mathrm{MPa}$. De acuerdo con lo anterior, se puede concluir que la resistencia de la unión puede ser incrementada hasta en un $36 \%$ a partir de la modificación de las variables de procesamiento de los recubrimientos. Igualmente, puede decirse que bajo las condiciones estudiadas la preparación de la superficie determinará la resistencia del sistema, siempre y cuando no existan otros problemas de procesamiento que disminuyan la cohesión entre capas de recubrimientos.

Los valores medidos de resistencia se encuentran en los límites superiores de los 
valores reportados para la técnica de aspersión por llama por varios autores [15], [16], lo cual indica un adecuado procesamiento de este tipo de recubrimientos.

Para el caso del recubrimiento $\mathrm{Ni}-2$ se observan los mayores valores de resistencia, para el caso de las superficies preparadas mediante alúmina y con temperatura de precalentamiento altas. En todos los casos se observaron diferencias significativas entre los niveles de preparación selec- cionados, siendo el efecto de la preparación de la superficie sobre la resistencia más marcado que el de la temperatura de precalentamiento. Los resultados indican que la resistencia puede ser incrementada hasta en un $61 \%$. Este resultado es importante porque indica que la resistencia de este recubrimiento es muy susceptible de ser mejorada a partir del procedimiento de aplicación.

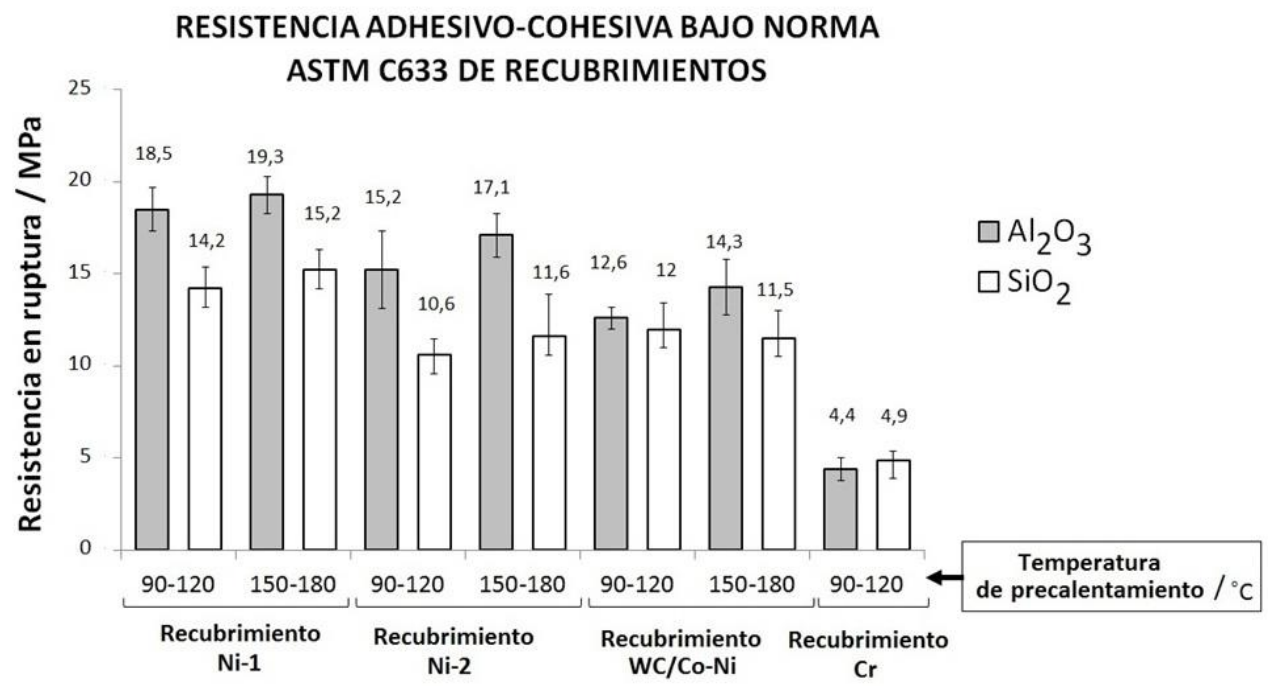

Fig. 6. Efecto de la preparación de la superficie y la temperatura de precalentamiento en la resistencia adhesivo-cohesiva de recubrimientos estudiados. Fuente: Autores

El recubrimiento WC/Co-Ni mostró los mayores valores de resistencia cuando las superficies son preparadas con alúmina y la temperatura de precalentamiento es alta. Los resultados indican que existen diferencias significativas entre los niveles de temperatura de precalentamiento seleccionados. De acuerdo con los valores de resistencia obtenidos, se puede concluir que la resistencia de la unión puede ser incrementada en un $25 \%$ con la modificación de los parámetros de procesamiento. Para este recubrimiento se apreciaron diferencias no significativas entre las 2 condiciones de preparación superficial para el caso de temperaturas de precalentamiento bajas. Este resultado está asociado con la generación de superficies con topografía superficial muy similar, después de aplicar la capa base de recubrimiento $\mathrm{Ni}^{-1}$ las cuales, para temperaturas de precalentamiento bajas, no generan modificaciones significativas en los valores de resistencia adhesivo-cohesiva.

Para el caso de la temperatura de precalentamiento alta existen diferencias significativas entre los niveles de preparación. La diferencia entre los valores de resistencia de las uniones está asociada a las pequeñas diferencias en rugosidad las cuales generan diferencias en el apilamiento de las laminillas cuando impactan la superficie. Las laminillas que impactan sobre superficies más rugosas finalmente permanecen con un estado de esfuerzos residuales menor y pueden resistir mayores esfuerzos en el ensayo de adherencia. 
Los resultados del recubrimiento $\mathrm{Cr}$ indican que la variación superficial tienen un efecto sobre la carga máxima soportada por los recubrimientos, sin embargo, este efecto no es significativo y solo incrementa la resistencia en $0,5 \mathrm{MPa}$ para los niveles estudiados. Esto indica que el incremento es tan solo del $11 \%$. Este comportamiento puede estar asociado con la generación de superficies muy similares después de la aplicación de la capa base, al igual que en el caso del recubrimiento $\mathrm{WC} / \mathrm{Co}-\mathrm{Ni}$.

\subsection{Discusión sobre el efecto de la tempera- tura de precalentamiento y el acabado superficial}

Uno de los principales objetivos de realizar un precalentamiento a las superficies es remover la humedad y las grasas que se encuentren allí. El precalentamiento debe ser realizado mediante una llama neutra con el fin de no modificar las superficies, la cual puede ser la misma de la pistola de aspersión térmica como se llevó a cabo en este trabajo. Las temperaturas recomendadas para realizar el precalentamiento se encuentran entre 100 y $200^{\circ} \mathrm{C}$. Las tensiones térmicas residuales durante el enfriamiento y durante el cambio de fase dependen de las temperaturas de precalentamiento, de la naturaleza de los materiales de la capa y el sustrato y de sus propiedades físicas (conductividad térmica y dilatación) [17], [18].

El precalentamiento favorece la reducción de las tensiones residuales internas que a su vez influencian la adherencia de las capas. Cuando las partículas chocan con un sustrato existe un enfriamiento muy rápido, el cual genera una contracción del material depositado, y entre más pequeña sea la diferencia de temperatura se genera un enfriamiento menos severo.

Los resultados experimentales muestran que la variación de la temperatura de precalentamiento en el rango estudiado no tiene un efecto significativo en la microestructura y la resistencia de los recubri- mientos estudiados. Para el recubrimiento $\mathrm{Ni}^{-} 1$ solo se reportó un aumento en la resistencia adhesivo-cohesiva de la unión entre 4 y $7 \%$, para el recubrimiento $\mathrm{Ni}^{-} 2$ este aumento fue de 9 a $13 \%$ y en el recubrimiento $\mathrm{WC} / \mathrm{Co}-\mathrm{Ni}$ se observaron incrementos entre un 4 y un $12 \%$. A pesar del resultado anterior, el control de la temperatura de precalentamiento es imperativo ya que si se exceden ciertos límites los recubrimientos crecerán con defectos no deseados, como por ejemplo desprendimientos en los bordes.

Por otro lado, el acabado superficial juega un papel determinante en la morfología final de las laminillas que resultan del impacto de partículas fundidas. Por un lado, sobre superficies más rugosas las partículas no se deforman completamente debido a una mayor fricción entre el líquido y la superficie sólida, pero al mismo tiempo se dan mejores condiciones de adherencia mecánica, así como la posibilidad de anular los esfuerzos generados durante el crecimiento del recubrimiento. En las muestras estudiadas en este trabajo, el acabado superficial tuvo una influencia más significativa que la temperatura de precalentamiento. Para el caso del recubrimiento $\mathrm{Ni}^{-1}$ el aumento estuvo entre $27-30 \%$, para el recubrimiento $\mathrm{Ni}-2$ estuvo entre $44-47 \%$, en el recubrimiento $\mathrm{WC} / \mathrm{Co}-\mathrm{Ni}$ osciló entre 5-25\%, y para el recubrimiento $\mathrm{Cr}$ el aumento fue del $10 \%$. Los recubrimientos sin capa base son muy sensibles a la variación del acabado superficial (en términos de resistencia adhesivo-cohesiva de la unión), mientras que la resistencia de los recubrimientos que requieren una capa base no es sensible a la modificación de este parámetro. Lo anterior no indica necesariamente que en términos prácticos el uso de diferentes abrasivos lleve a la obtención de recubrimientos similares cuando se usa capa base, pues fue observado, por ejemplo, que cuando se superan levemente los valores de temperatura de precalentamiento requeridos, los acabados con alúmina son mejores que con sílice para garantizar la 
fijación de las capas, aún en el caso en que se tenía capa base.

\subsection{Análisis estadístico de resultados de resistencia adhesivo-cohesiva}

Se diseñó un experimento con solo dos factores y se llevó a cabo el correspondiente análisis de varianza, así como la comparación de medias de tratamiento con un control [14]. Se tienen dos factores (integridad superficial y temperatura de precalentamiento) con 2 niveles, la variable de salida es resistencia adhesivo-cohesiva de la unión. Las hipótesis apropiadas son:

$\mathrm{H} 0=\mu 1=\mu 2=\mu 3$

$\mathrm{HA}=$ No se cumple H0

La relación utilizada para rechazar la hipótesis Ho fue:

$$
F_{0}>F_{\alpha, a-1, N-a}
$$

El valor de F se tomó de la tabla "Puntos porcentuales de la distribución $F^{v '[19] . ~}$

La Tabla 4 muestra los resultados utilizados para comprobar el modelo estadístico, así como los resultados de análisis de varianza. De la tabla "Puntos porcentuales de la distribución $F^{v " ~[19], ~ s e ~ o b t u v o ~ q u e ~}$ $\mathrm{F} 0.1, \mathrm{a}-1, \mathrm{~N}-\mathrm{a} .=2.6$. La hipótesis todas las medias de los tratamientos son iguales se rechaza porque el valor de Fo es mayor a 2,6 para el caso de los recubrimientos $\mathrm{Ni}-1$ y $\mathrm{Ni}-2$, lo cual indica estadísticamente que existe una diferencia entre los tratamientos. De acuerdo con lo anterior, la rugosidad superficial es el parámetro que influye más significativamente en la resistencia adhesivo-cohesiva. Para el caso del recubrimiento $\mathrm{WC} / \mathrm{Co}^{-} \mathrm{Ni}$ no existieron diferencias estadísticas significativas, pero se recomienda utilizar los parámetros que reportaron los valores de resistencia adhesivo-cohesiva más altos. El análisis estadístico coincide con los resultados observados anteriormente en las gráficas de resistencia contra métodos de preparación de la superficie.

\subsection{Análisis de las superficies de falla}

En esta sección se realizará un análisis de las superficies de falla de los recubrimientos $\mathrm{Ni}^{-1}$ y WC/Co-Ni dado que, por sus altos valores de resistencia adhesivocohesiva, son importantes tecnológicamente para aplicaciones de protección de elementos de máquinas. Los resultados de los recubrimientos $\mathrm{Ni}^{-2}$ y $\mathrm{Cr}$ pueden ser consultados en otras fuentes bibliográficas [20].

Las Figs. 7a, b y c muestran las fallas típicas de los recubrimientos $\mathrm{Ni}^{-1}$ en función de la preparación. A pesar de que la falla fue en general de tipo adhesivo como se muestra en la Fig. 7a, se presentaron también algunos casos de fallas mixtas en las que, en principio, no es posible concluir sobre su inicio en el adhesivo o en el recubrimiento. Sin embargo, como los datos de este tipo de fallas se encuentran dentro de la desviación estándar de las fallas 100\% adhesivas se presume que su origen fue en el recubrimiento. La Fig. 7b muestra una imagen de las fallas obtenida mediante microscopía electrónica de barrido, allí se observa que efectivamente las fallas son del tipo adhesivo al analizarse la superficie del sustrato. La inspección de los residuos de recubrimiento sobre las superficies reveló que las fallas se propagaron entre las laminillas evidenciando una adecuada cohesión entre las mismas. En algunos casos se observan vacíos generados por partículas sin fundir y salpicaduras. En algunas muestras se presentaron fallas del tipo cohesivo en la resina o adhesivo en la intercara resina-contracuerpo utilizada, las cuales no fueron tenidas en cuenta al realizar el promedio de los datos obtenidos y solo se puede concluir que en esas pruebas la resistencia de la unión es superior al valor de ruptura del adhesivo. Es importante notar que en todos los casos los resultados presentados son el promedio de al menos 3 ensayos válidos. 
Microestructura y resistencia adhesivo-cohesiva de recubrimientos de níquel, carburo de tungsteno y óxido de cromo aplicados por aspersión térmica

Tabla 4. Resultados del análisis de varianza. Fuente: Autores

\begin{tabular}{|c|c|c|c|c|c|}
\hline \multirow{2}{*}{$\begin{array}{c}\text { Recubrimiento } \\
\mathrm{Ni}-1\end{array}$} & \multirow[b]{2}{*}{ SSt } & \multirow{2}{*}{$\begin{array}{c}\text { SUMA } \\
106,5\end{array}$} & \multirow[t]{2}{*}{ LIBERTAD } & & \multirow[b]{2}{*}{$\mathrm{Fo}$} \\
\hline & & & & & \\
\hline $\begin{array}{l}\text { Temperatura de } \\
\text { precalentamiento }\end{array}$ & SS TEMP & 4,3 & 1,0 & 4,3 & 0,3 \\
\hline $\begin{array}{l}\text { Rugosidad } \\
\text { superficial }\end{array}$ & SS RUGOSIDAD & 88,4 & 1,0 & 88,4 & 6,4 \\
\hline Interacción & S INTERACCION & 0,0 & 1,0 & 0,0 & 0,0 \\
\hline Error & Sse & 13,7 & 16,0 & 0,9 & \\
\hline Total & & 106,5 & 19,0 & & \\
\hline Recubrimiento & & SUMA & LIBERTAD & & \\
\hline $\mathrm{Ni}-2$ & SSt & 187,8 & & & Fo \\
\hline $\begin{array}{l}\text { Temperatura de } \\
\text { precalentamiento }\end{array}$ & SS TEMP & 10,8 & 1,0 & 10,8 & 0,2 \\
\hline $\begin{array}{l}\text { Rugosidad } \\
\text { superficial }\end{array}$ & SS RUGOSIDAD & 128,3 & 1,0 & 128,3 & 2,7 \\
\hline Interacción & S INTERACCION & 1,1 & 1,0 & 1,1 & 0,0 \\
\hline Error & Sse & 47,6 & 16,0 & 3,0 & \\
\hline Total & & 187,8 & 19,0 & & \\
\hline Recubrimiento & & SUMA & LIBERTAD & & \\
\hline $\mathrm{WC} / \mathrm{Co}-\mathrm{Ni}$ & SSt & 47,6 & & & Fo \\
\hline $\begin{array}{l}\text { Temperatura de } \\
\text { precalentamiento }\end{array}$ & SS TEMP & 2,0 & 1,0 & 2,0 & 0,1 \\
\hline $\begin{array}{l}\text { Rugosidad } \\
\text { superficial }\end{array}$ & SS RUGOSIDAD & 14,2 & 1,0 & 14,2 & 0,5 \\
\hline Interacción & S INTERACCION & 4,9 & 1,0 & 4,9 & 0,2 \\
\hline Error & Sse & 26,6 & 16,0 & 1,7 & \\
\hline Total & & 47,6 & 19,0 & & \\
\hline
\end{tabular}

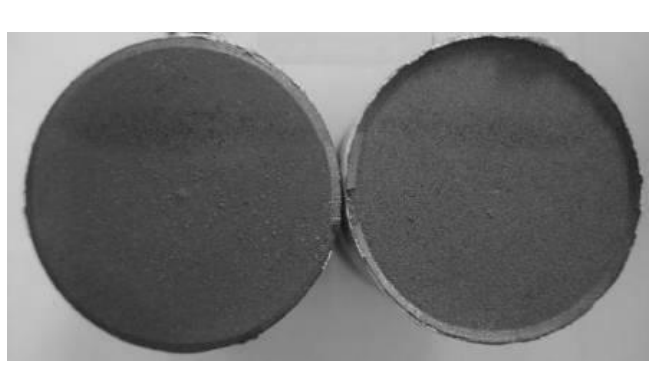

a) $\mathrm{Ni}-1 . \mathrm{ME}$

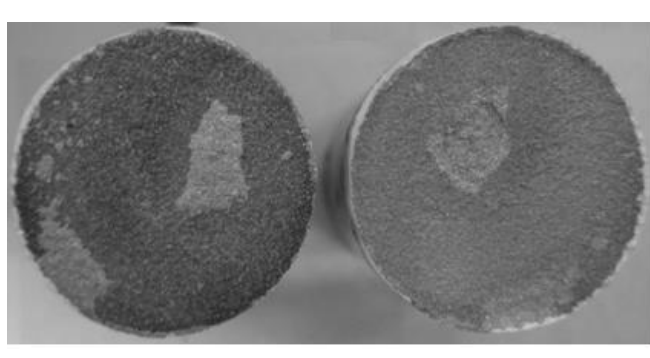

d) $\mathrm{WC} / \mathrm{Co}-\mathrm{Ni}$. ME

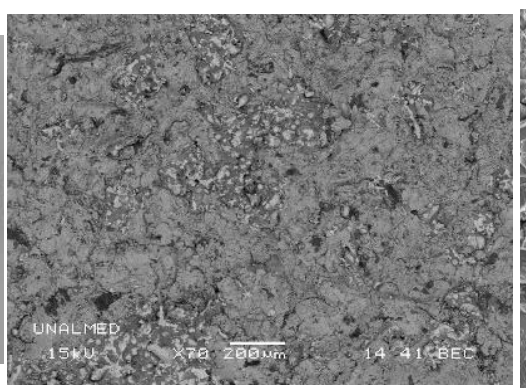

b) $\mathrm{Ni}-1$. MEB

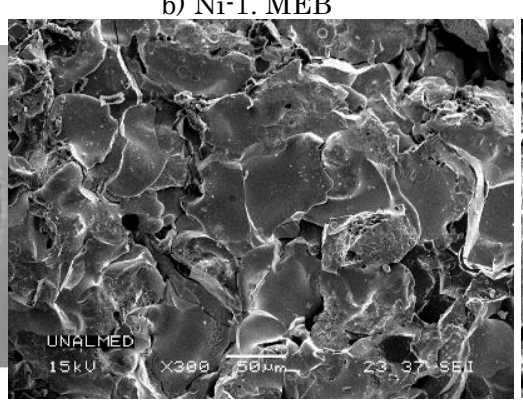

e) $\mathrm{WC} / \mathrm{Co}-\mathrm{Ni}$. MEB

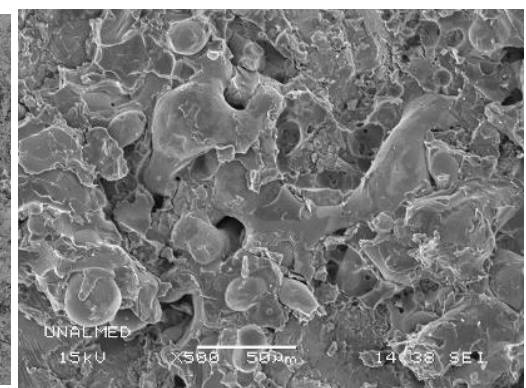

c) $\mathrm{Ni}-1 . \mathrm{MEB}$

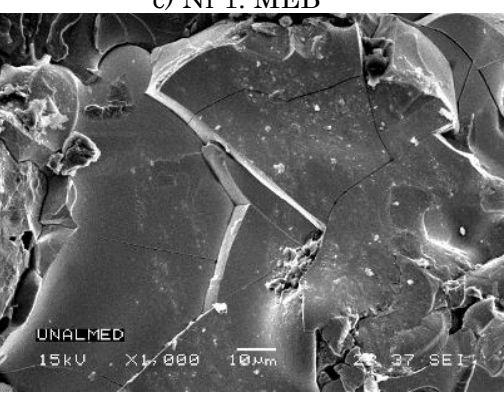

f) $\mathrm{WC} / \mathrm{Co}-\mathrm{Ni}$. MEB

Fig. 7. Aspecto típico de las superficies de falla después de ensayo ASTM C633 en microscopio estereoscópico (ME) y electrónico de barrido (MEB). Fuente: Autores 
Las Figs. 7c, d y e muestran las fallas típicas de los recubrimientos $\mathrm{WC} / \mathrm{Co}-\mathrm{Ni}$. Se observaron dos tipos de fallas fundamentalmente: fallas adhesivas internas y fallas cohesivas en la capa resistente. La inspección de estas superficies de falla mediante MEB evidenció que las fallas se propagan al interior de las laminillas del recubrimiento. Las laminillas mostraron fractura frágil como se puede observar en la Fig. 7e. Las fallas adhesivas internas se observaron en muestras preparadas con temperatura de precalentamiento baja y se presentan en la Fig. 7c, donde se observa el aspecto general de las superficies de fractura de probetas en el cuerpo y contracuerpo correspondientes a la misma probeta. La relación con la microestructura muestra que los mayores valores de resistencia adhesivo-cohesiva coinciden con los valores más bajos de porosidad.

\section{CONCLUSIONES}

En la aplicación de recubrimientos por aspersión térmica por combustión sobre acero inoxidable ASTM A743 grado CA6NM se mostró que variaciones en la preparación superficial tienen efecto significativo y reproducible sobre la resistencia adhesivo-cohesiva de diversos sistemas estudiados.

La rugosidad superficial es el parámetro que influye más significativamente en la resistencia adhesivo-cohesiva, aumentando entre un $36 \%$ y un $51 \%$ la adherencia de los recubrimientos $\mathrm{Ni}^{-1}$ y Ni-2, respectivamente. Su efecto sobre la adherencia de los recubrimientos $\mathrm{WC} / \mathrm{Co}-\mathrm{Ni}$ y $\mathrm{Cr}$ fue menos relevante.

Los recubrimientos identificados como $\mathrm{Ni}^{-1}$ y Ni-2 presentaron los mayores valores de resistencia adhesivo-cohesiva, lo cual es consistente con su aplicación típica como capas de anclaje en diversas aplicaciones industriales. El recubrimiento $\mathrm{WC} / \mathrm{Co}^{-} \mathrm{Ni}$ presentó también un buen com- portamiento, mientras que el recubrimiento $\mathrm{Cr}$ ofreció los valores más bajos.

Fue identificado también un efecto de la temperatura de precalentamiento sobre la resistencia adhesivo-cohesiva de los recubrimientos, pero su significancia fue menor que la observada para la preparación de las superficies. En términos generales, el incremento varió entre $4 \%$ y $12 \%$ para distintos recubrimientos.

Las fallas observadas, luego de las pruebas de resistencia adhesivo-cohesiva, fueron dominantemente de carácter adhesivo, lo cual reflejó una buena cohesión en los recubrimientos y permitió establecer correlaciones con las condiciones de aplicación.

\section{AGRADECIMIENTOS}

Los autores agradecen a Empresas Públicas de Medellín (EPM) e industrias HRV por la disposición de su infraestructura física, y a los laboratorios de Metalografía y Soldadura de la Universidad Nacional de Colombia, por su colaboración en las labores experimentales.

\section{REFERENCIAS}

[1] D. Crawner, "Thermal Spray Processes, Handbook of Thermal Spray Technology, J," Int. ASM, 2004.

[2] I. M. Hutchings, "Tribology: friction and wear of engineering materials," 1992.

[3] K.-H. Zum Gahr, Microstructure and wear of materials, vol. 10. Elsevier, 1987.

[4] J. R. Davis, Handbook of Thermal Spray Technology. ASM International, 2004.

[5] C. Salinas, J. J. Coronado, and G. Latorre, "Evaluación de la multicapa $\mathrm{Ni}, \mathrm{Al}, \mathrm{Mo} / \mathrm{Al} 2 \mathrm{O} 3 \mathrm{TiO} 2$, aplicada por el proceso de rociado térmico por 
combustión," Rev. Tecnura, vol. 8, no. 16, pp. 15-25, 2005.

[6] R. S. C. Paredes, S. C. Amico, and a. S. C. M. D'Oliveira, "The effect of roughness and pre-heating of the substrate on the morphology of aluminium coatings deposited by thermal spraying," Surf. Coatings Technol., vol. 200, no. 9, pp. 30493055, 2006.

[7] M. Mellali, P. Fauchais, and A. Grimaud, "Influence of substrate roughness and temperature on the adhesion/cohesion of alumina coatings," Surf. Coatings Technol., vol. 81, no. 2-3, pp. 275-286, 1996.

[8] P. Fauchais, A. Vardelle, M. Vardelle, and M. Fukumoto, "Knowledge concerning splat formation: an invited review," $J$. Therm. Spray Technol., vol. 13, no. 3, pp. 337-360, 2004.

[9] A. Nistal, E. García, C. GarcíaDiego, M. I. Osendi, and P. Miranzo, "Flame spraying of adherent silicon coatings on SiC substrates," Surf. Coatings Technol., vol. 270, pp. 815, 2015.

[10] L. Jin, G. Liu, P. Li, H. Zhou, C. Wang, and G. Zhou, "Adhesion strength and thermal shock properties of nanostructured 5La3TiYSZ, 8LaYSZ and 8CeYSZ coatings prepared by atmospheric plasma spraying," Ceram. Int., vol. 41, no. 9, pp. 12099-12106, 2015.

[11] ASTM, "ASTM NORMA C633. Standard guide to testing adhesion/cohesion of thermal spray coatings." p. 13, 2001.

[12] A. Toro, "Informe de Proyecto de investigación EPM-UN-COLCIE," Medellin, 2005.
[13] J. D. Osorio, H. P. Gómez, and A. Toro, "Efecto del tratamiento térmico post soldadura sobre la microestructura del acero inoxidable grado ca6nm para la construcción de rodetes hidráulicos," Sci. Tech., vol. 1, no. 36, 2007.

[14] J. D. Crawford, "CA6NM an update," in 29th Annual Steel Founder's Society of America Techical and Operating Conference, 1974, pp. 113.

[15] J. F. Santa, J. C. Baena, and A. Toro, "Slurry erosion of thermal spray coatings and stainless steels for hydraulic machinery," Wear, vol. 263, no. 1, pp. 258-264, 2007.

[16] R. C. Tucker Jr, "On the relationship between the microstructure and the wear characteristics of selected thermal spray coatings," Therm. Spraying--Current Status Futur. Trends., vol. 1, pp. 477-482, 1995.

[17] P. Araujo, D. Chicot, M. Staia, and J. Lesage, "Residual stresses and adhesion of thermal spray coatings," Surf. Eng., vol. 21, no. 1, pp. 35-40, 2005.

[18] J. H. Lindsay, "Coatings and coating processes for metals," ASM Int. Member/Customer Serv. Center, Mater. Park. OH 44073-0002, USA, 1998. 353, 1998.

[19] D. C. Montgomery, D. C. Montgomery, and D. C. Montgomery, Design and analysis of experiments, vol. 7. Wiley New York, 1984.

[20] J. F. Santa, "Adherencia y resistencia al desgaste erosivo de recubrimientos por aspersión térmica," Universidad Nacional de Colombia, 2008. 